Importance of the volcano rabbit (Romerolagus diazi) in the coyote

(Canis lastrans) and bobcat (Lynx rufus) diets

\title{
Importancia del conejo zacatuche (Romerolagus diazi) en la dieta del coyote (Canis lastrans) y del lince (Lynx rufus)
}

\author{
Juan Manuel Uriostegui-Velarde ${ }^{1}$, Zuri Samuel Vera-García ${ }^{1}$, Luis Gerardo Ávila-Torresagatón ${ }^{1}$ Areli Rizo-Aguilar ${ }^{1}$, \\ Mircea G. Hidalgo-Mihart ${ }^{2}$ y José Antonio Guerrero ${ }^{1^{*}}$
}

\begin{abstract}
${ }^{1}$ Facultad de Ciencias Biológicas, Universidad Autónoma del Estado de Morelos. Avenida Universidad 1001, Cuernavaca 62210. Morelos, México. E-mail: uvjm@hotmail.com (JMUV) samuel_vera65@hotmail.com (ZSVG), luis.avila@uaem.mx (LGAT), areli.rizo@ uaem.mx (ARA), aguerrero@uaem.mx (JAG)

${ }^{2}$ División Académica de Ciencias Biológicas, Universidad Juárez Autónoma de Tabasco. Km 0.5 carretera Villahermosa-Cárdenas, Villahermosa 86039. Tabasco, México. E-mail: mhidalgo@yahoo.com (MHM)

${ }^{*}$ Corresponding author
\end{abstract}

Introduction: Predation may have important effects on prey populations, especially when the prey species is considered endangered such as the volcano rabbit (Romerolagus diazi). Understanding the importance of the volcano rabbit in the coyote and bobcat diet is important to recognize the possible impact that thesepredators can have on the populations of this endangered rabbit.

Methods: We studied the food habits of the coyote and during three climate seasons in the Chichinautzin Biological Corridor through scat analysis. We obtained the frequency of occurrence and consumed biomass of each identified prey item. Additionally we estimated the relative abundance of some of the potential prey species for these two predators and analyzed the relationship between prey abundance and the use of the prey found on the diet of these two predators.

Results: The most common prey species and with the greatest biomass contribution on the diet of coyotes and bobcats were the rabbits of the genus Sylvilagus sp., followed by the volcano rabbit. We did not find differences in the frequency of occurrence of prey or in the consumed biomass between the studied seasons, indicating that the diet of both predators remains constant throughout the year. Prey selection analysis showed that both predators selected Sylvilagus rabbits, while volcano rabbits are consumed less than their availability in the environment.

Discussion and conclusions: Reduced consumption of the volcano rabbit according with their availability on the environment by coyotes and bobcats could be the result of the selection of larger prey and the nocturnal behavior patterns of both predators. Even though we did not find that both predators are selecting volcano rabbits on their diets, our results showed that coyotes and bobcats are important predators for $R$. diazi. It is necessary to determine the potential number of volcano rabbits consumed by both predators in order to understand the impact of these predators on this endangered species.

Key words: conservation; endangered species; predation; prey selection; volcano rabbit.

Introducción: La depredación puede tener efectos importantes en las poblaciones de las presas, siendo este efecto mayor cuando la presa es una especie en peligro de extinción. Entender la importancia del conejo zacatuche en la dieta del coyote y del lince es importante para conocer el impacto que estos depredadores pueden estar ejerciendo sobre las poblaciones de este conejo catalogado en peligro de extinción.

Metodología: Se estudió la dieta del coyote y el lince durante tres temporadas climáticas en el Corredor Biológico Chichinautzin a través del análisis de sus heces fecales. Calculamos la proporción de aparición y el porcentaje de biomasa total consumida de cada presa identificada. Además, se estimó la abundancia relativa de las presas potenciales en el ambiente y se analizó la relación entre las abundancias de predadores con la presencia de presas que son componentes de sus dietas. 
Resultados: La presa con mayor frecuencia de ocurrencia y la que mayor biomasa aporta a la dieta de ambos depredadores fue Sylvilagus sp., seguida por Romerolagus diazi. No se encontraron diferencias significativas en la frecuencia de ocurrencia de las presas, ni para la proporción de biomasa consumida entre las diferentes temporadas analizadas. Este resultado indica que la dieta de ambos depredadores se mantiene constante durante el año. El análisis de selección de presas mostró que ambos depredadores seleccionan a conejos del género Sylvilagus, mientras que los zacatuches son consumidos en menor proporción que su disponibilidad en el ambiente.

Discusión y conclusiones: El menor consumo de zacatuches por el coyote y el lince de acuerdo a su disponibilidad en el ambiente podría ser el resultado de la selección de presas de mayor tamaño y a los patrones de comportamiento mayormente nocturnos de ambos depredadores. A pesar de que no se encontró selección hacia los zacatuches por parte de coyotes y linces, los resultados mostraron que los coyotes y linces son depredadores importantes de $R$. diazi. Es necesario determinar a través de estudios específicos el número potencial de zacatuches consumidos por los depredadores y su impacto en las poblaciones de la especie.

Palabras clave: conservación; depredación; especie amenazada; selección de presas; zacatuche.

\section{Introducción}

La depredación puede tener efectos serios en las poblaciones de las presas (Nilsson 2001; Vucetich et al. 2005; Gervasi et al. 2012). En ambientes estables, las interacciones depredador-presa se mantienen constantes (Begon et al. 1996). Sin embargo, cuando un ecosistema sufre modificaciones en un periodo de tiempo corto, pueden ocurrir cambios en el tipo y la abundancia de los depredadores, lo que puede afectar a las especies presa de manera directa con su muerte o bien con cambios en dicha interacción (Sih et al. 1998; Gervasi et al. 2013). En consecuencia, puede ocurrir que las especies presa adopten nuevas estrategias que le permitan reducir el riesgo de depredación (Shapira et al. 2008), o bien que ocurra un aumento en la tasa de depredación, lo que resultará en una drástica reducción de los tamaños poblacionales de las presas (Estes et al. 1998; Vucetich et al. 2005; Festa-Bianchet et al. 2006).

Este efecto es particularmente importante cuando la presa es una especie en peligro de extinción (Drewien et al. 1985; Crawford et al. 2010). Los casos mejor documentados en este sentido han sido los depredadores introducidos, los cuales han causado la extinción de especies presa, particularmente en islas (Hanna y Cardillo 2014). Este efecto también se ha documentado con depredadores nativos, los cuales han sido favorecidos debido a cambios en el ambiente producidos por el hombre, de tal forma que el impacto sobre las presas ha sido mayor al esperado en un ambiente no modificando, colocando en riesgo de extinción a la especie presa (Crawford et al. 2010; Ripple et al. 2013).

El conejo zacatuche (Romerolagus diazi) es una especie endémica de las montañas centrales del Eje Volcánico Tranversal. Sus poblaciones se concentran en cuatro áreas núcleo y 12 periféricas, ubicadas en los Volcanes Pelado, Tláloc, Popocatépetl e Iztaccíhuatl, en altitudes por arriba de los 2,800 m (Velázquez et al. 1996; Rizo-Aguilar et al. 2015). Es una especie especialista de hábitat porque su sobrevivencia depende de la presencia de pastizales amacollados de los géneros Mulhenbergia, Festuca y Jarava (Velázquez 1994). Es una especie abundante en las zonas donde esos pastizales tienen un cobertura densa y mayor altura, prácticamente está ausente fuera de estas zonas (Rizo-Aguilar et al. 2015). El hábitat del zacatuche ha disminuido drásticamente como resultado de actividades humanas como la agricultura, el pastoreo y extracción de tierra para jardinería (Portales et al. 1997; Velázquez et al. 2011), además de que se ha documentado que enfrenta amenazas importantes derivadas del cambio climático 
(Anderson et al. 2009). Debido a estos factores, el conejo zacatuche está considerado en peligro de extinción en la Norma Oficial Mexicana NOM-059-SEMARNAT-2010 (SEMARNAT 2010), así como por la Unión Internacional para la Conservación de la Naturaleza (AMCELA et al. 2008).

El zacatuche es depredado naturalmente por coyotes, linces y comadrejas, reptiles como las serpientes de cascabel y aves rapaces (Cervantes y Martínez-Vázquez 1996). Se ha encontrado que en aquellas zonas donde el hábitat es adecuado para el zacatuche, es posible encontrar poblaciones abundantes de este lagomorfo, de tal forma que en el caso particular de la dieta de linces y coyotes. El zacatuche es una de las primeras presas más consumida por estos depredadores en la zona del Ajusco, con importancia comparable a la de otros conejos de mayor tamaño como Sylvilagus floridanus y S. cunicularius (Romero 1993; Aranda et al. 1995, 2002), mientras que en la zona del Iztaccíhuatl-Popocatépetl es la cuarta más consumida (Martínez-García et al. 2014). Además, se ha mencionado que los perros ferales pueden ser importantes depredadores de la especie (Portales et al. 1997).

En una especie con distribución limitada y sujeta a grandes presiones antropogénicas como $R$. diazi, el efecto de la depredación natural podría ser importante y considerarse como una amenaza más a su supervivencia. El entender la importancia del conejo zacatuche en la dieta del coyote y del lince nos permitirá conocer el efecto potencial que ambos depredadores potencialmente podrían ejercer sobre las poblaciones de este lagomorfo. Si existiera una selección del zacatuche sobre la disponibilidad de los mismos en el ambiente indicaría que los depredadores los prefieren sobre otras fuentes de alimento. Es así que para entender el efecto de los linces y coyotes podrían tener sobre el zacatuche analizamos los hábitos alimentarios de estos depredadores durante tres temporadas climáticas de un ciclo anual y comparamos los resultados con la abundancia relativa de presas potenciales en el ambiente.

\section{Materiales y métodos}

Área de estudio. El estudio se llevó a cabo dentro del Área de Protección de Flora y Fauna Corredor Biológico Chichinautzin (COBIOCH) ubicada al norte del estado de Morelos. Específicamente trabajamos en los alrededores del Cerro San Ignacio, en la zona Centro-Norte de la Fracción I del COBIOCH (Figura 1). En el área hay distintas asociaciones vegetales las cuales consisten de bosque de pino-encino, bosque de pino, bosque de Abies y zacatonales subalpinos (Velázquez y Cleef 1993). La altitud esta entre los 2,900 y 3,200 m; el clima predominante es templado semifrío y una temperatura media anual de entre 5 y $12{ }^{\circ} \mathrm{C}$ (Contreras-MacBeath et al. 2004). En el área se presentan tres temporadas climáticas derivadas de la presencia de heladas y lluvias (seca fría, seca cálida y lluviosa).

Colecta de heces fecales y análisis de la dieta. La dieta del coyote y lince se estudió por medio del análisis de sus heces fecales (Klare et al. 2011). Entre junio de 2008 y mayo de 2009 se recorrieron mensualmente a pie aproximadamente $20 \mathrm{~km}$ de caminos y brechas en el área de estudio en búsqueda de heces fecales de estos depredadores. Un mes antes del inicio de esta búsqueda, el área se recorrió y se eliminaron todas las heces fecales, de tal forma que las heces fecales colectadas solo correspondían al mes en curso. Las heces fecales fueron identificadas en campo basándose en la forma, tamaño, color y olor, pero sobre todo la presencia de huellas asociadas (Aranda 


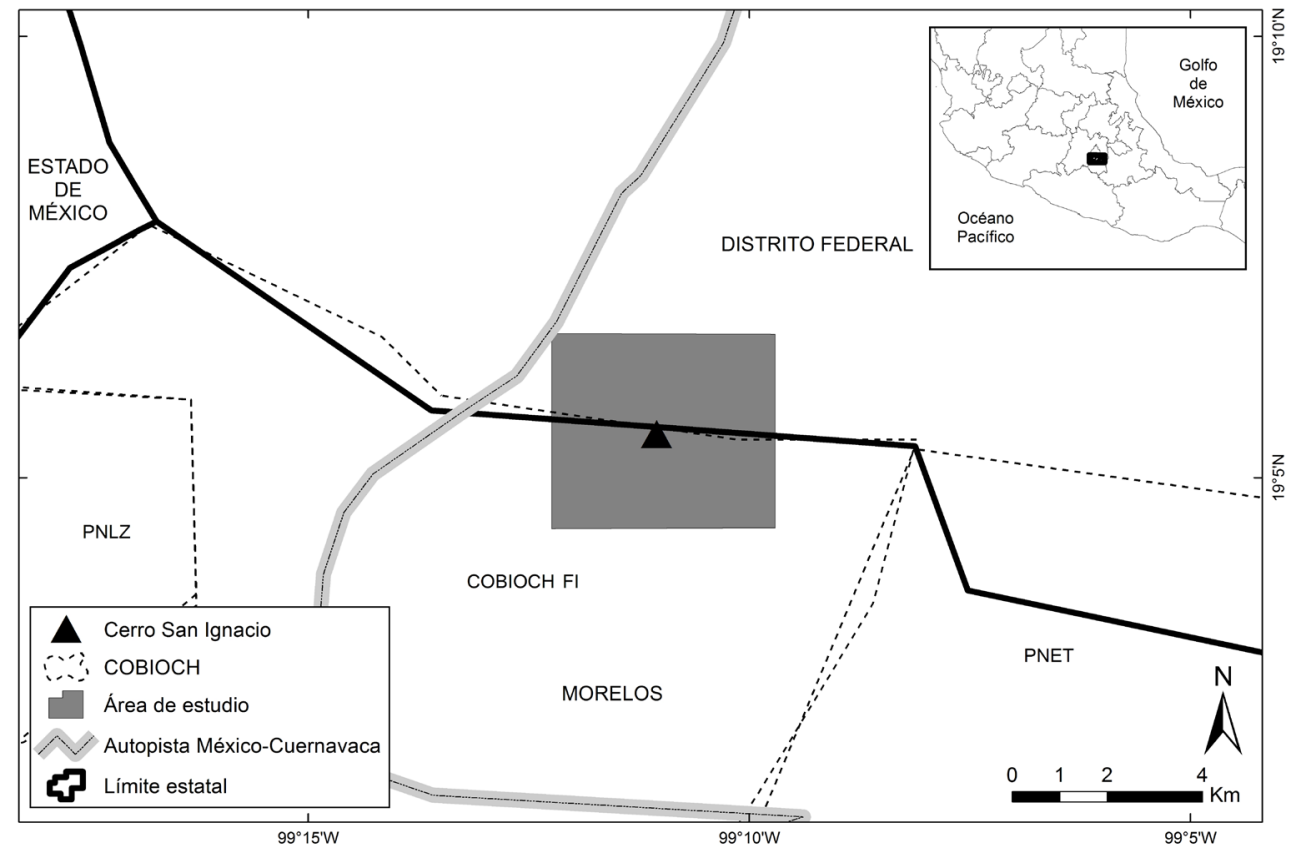

Figura 1. Esquema del área de estudio dentro de las Áreas Naturales Protegidas que conforman el $\mathrm{COBIOCH}$ PNLZ Parque Nacional Lagunas de Zempoala, PNET Parque Nacional El Tepozteco, COBIOCH FI y FII, Fracciones I y II del Corredor Biológico Chichinautzin.

2000). Las heces fecales halladas en un avanzado estado de desintegración y aquellas que no pudieron ser identificadas por sus características o por la ausencia de huellas asociadas no fueron recolectadas. Posterior a la recolección en campo, las heces fecales fueron transportadas en bolsas de plástico y procesadas en el laboratorio siguiendo la metodología estándar (Klare et al. 2011).

La identificación de las presas fue realizada al separar los diferentes componentes de las heces fecales (pelos de mamíferos, restos de artrópodos, plumas y materia vegetal). Para el análisis de la dieta sólo se consideraron los pelos de mamíferos debido a que representaban más del $95 \%$ de la dieta de ambos depredadores y porque el análisis de selectividad se enfocó a este grupo de presas. De cada hez fecal se seleccionaron 15 pelos, los cuales fueron identificados por medio de un análisis microscópico de sus patrones medulares tomando como referencia claves y guías de identificación (Monroy-Vilchis y Rubio-Rodríguez 2003). Se realizó también una comparación con pelos obtenidos de especies capturadas en campo y de ejemplares de la Colección de Mamíferos del Centro de Investigación en Biodiversidad y Conservación (CMC) de la Universidad Autónoma del Estado de Morelos. Los pelos fueron identificados hasta el nivel taxonómico de especie cuando fue posible y asignados a una presa en particular.

Para la caracterización y el análisis de la dieta las heces fecales se agruparon en tres temporadas del año que correspondieron a la época de lluvias (junio a septiembre), seca fría (octubre a enero) y seca cálida (febrero a mayo). Se estimó la frecuencia de ocurrencia (FO) para cada una de estas temporadas de los distintos componentes identificados de la dieta (Ackerman et al. 1984) y el porcentaje de biomasa total consumida (BTC; Klare et al. 2011). En el caso del coyote, la estimación de la biomasa consumida se hizo usando el modelo de regresión lineal $(y=0.439+$ $0.008 x$ ) desarrollado para el lobo (Canis lupus) por Weaver (1993). En el lince se utilizó el modelo de regresión $(y=15.06+1.330 x)$ propuesto por Rühe et al. (2007) para 
el lince euroasiático (Lynx lynx). En ambas ecuaciones lineales, y representa la masa (en g) de la presa en cada hez fecal colectada, y $x$ representa la masa corporal (en g) de las presas (Weaver 1993). El peso de las especies presa se obtuvo a partir de las capturas realizadas en campo, calculando el promedio para cada una (Peromyscus sp., $n=6$; Neotomodon alstoni, $n=105$; Reithrodontomys sp., $n=18$; Sigmodon sp., $n=6$ y Sorex sp., $n=3$ ). Para aquellas especies presa que no se capturaron en campo, el peso consignado se tomó de las descripciones de las especies en la obra de Ceballos y Oliva (2005). Una vez estimados los valores de FO y el porcentaje de BTC, se llevó a cabo una prueba de independencia de $G$ agrupando los datos para los lagomorfos y para los roedores y soricomorfos para analizar si existían diferencias en estos parámetros de la dieta del lince y del coyote entre las tres temporadas (Sokal y Rohlf 1980) empleando el programa Statistica 8 (Statsoft 2007).

Disponibilidad de las presas. Para analizar la importancia del conejo zacatuche y de otras presas potenciales en la dieta del coyote y del lince se estimóla abundancia relativa de lagomorfos, roedores y soricomorfos como una medida de su disponibilidad en el ambiente (Litvaitis et al. 1996). La disponibilidad de presas se limitó a la estimación de la abundancia relativa de estos tres grupos de mamíferos debido a que todos los trabajos realizados en la región sobre la dieta del coyote y del lince muestran que son las presas de mayor consumo para estos depredadores (Romero 1993; Aranda et al. 1995; Aranda et al. 2002; Martínez-García et al. 2014).

Disponibilidad de lagomorfos. Se seleccionaron cuatro sitios de muestreo (de aproximadamente cuatro hectáreas cada uno) distribuidos en el área de estudio y tratando de incluir los distintos hábitats naturales. En cada sitio se colocó una gradilla de 25 parcelas de $1 \mathrm{~m}^{2}$ distribuidas en cinco líneas paralelas de cinco parcelas cada una. Cada línea estaba separada por $25 \mathrm{~m}$, mientras que las parcelas en las líneas estaban ubicadas cada $10 \mathrm{~m}$. El centro de las parcelas se marcó con una estaca de madera. Para los lagomorfos se utilizó el método de conteo de heces fecales como una medida de su abundancia relativa (Palomares 2001). Los excrementos del zacatuche son fácilmente distinguibles de las especies de Sylvilagus por su forma discoidal, hinchadas en la parte central y con un tamaño de 5 a $9 \mathrm{~mm}$ (Cervantes y MartínezVázquez 1996). Para cada muestreo, las parcelas eran limpiadas eliminando todas las heces fecales y aproximadamente un mes después se recolectaban y contaban todas las heces fecales encontradas dentro de cada parcela. Con los datos de conteos de heces fecales se calcularon índices de abundancia relativa de lagomorfos (IAR-L) para cada temporada climática. Para los lagomorfos se dividió el número de heces fecales colectadas en todos los cuadrantes entre el número total de cuadrantes, obteniendo así el número de heces fecales por metro cuadrado (heces fecales $/ \mathrm{m}^{2}$ ).

Disponibilidad de roedores y soricomorfos. Se realizaron capturas de roedores y soricomorfos dos noches consecutivas para cada una de las temporadas climáticas estudiadas, colocando una trampa Sherman en cada una de las 250 parcelas utilizadas para estimar la abundancia de los lagomorfos. Los individuos capturados se identificaron a nivel de especie o género usando guías regionales de mamíferos (Ceballos y Galindo-Leal 1984) y así como las descripciones y claves de Hall (1981). Una vez identificados los roedores y soricomorfos, los individuos capturados fueron liberados en el mismo sitio donde se capturaron. Con los datos de capturas de roedores y soricomorfos se calcularon los índices de abundancia relativa de roedores y soricomorfos (IAR-RS) para cada temporada, dividiendo el número de capturas de cada especie entre el número de trampas empleadas para cada temporada muestreada. 
Análisis de selección de presas. Para analizar si el conejo zacatuche es consumido como presa en la dieta del coyote y del lince de manera más o menos frecuente de lo esperado debido a su disponibilidad, se utilizó el método propuesto por Neu et al. (1974) y Byers et al. (1984), el cual combina una comparación inicial de $X^{2}$ seguida del cálculo de los intervalos de confianza de Bonferroni (Litvaitis et al. 1996). La prueba de $X^{2}$ se usa para determinar si existe una diferencia significativa entre el uso esperado que sería representado por la disponibilidad de las presas en el área (en nuestro estudio, la abundancia relativa de cada especie presa) y el uso observado, medido por el aporte de la biomasa de las especies presas en la dieta de ambos depredadores. Se utilizó el porcentaje de biomasa consumida para no sobrestimar la importancia de las presas pequeñas en la dieta de ambos carnívoros (Klare et al. 2011). Los análisis de $X^{2}$ y la estimación de los intervalos de confianza se realizaron de manera independiente para los lagomorfos y para los roedores y soricomorfos debido a que utilizamos dos métodos diferentes para estimar la abundancia relativa de ambos grupos. Los intervalos de confianza de Bonferroni se usan cuando la prueba de $X^{2}$ muestra que hay diferencia entre el uso esperado y el observado, con la finalidad de determinar qué presas están siendo seleccionadas (cuando el uso observado está por arriba del intervalo calculado y se presenta con signo positivo), evitadas (cuando el uso observado está por abajo del intervalo calculado y se presenta con signo negativo) o usadas de acuerdo a su disponibilidad en el ambiente (cuando el uso observado cae dentro del intervalo estimado y no presenta signo positivo ni negativo; Byers et al. 1984). Este método se usó anteriormente para evaluar la selección de distintos tipos de hábitat por el zacatuche (Velázquez y Heil 1996).

\section{Resultados}

Composición de la dieta. Se analizaron 119 heces fecales de coyote (lluvia = 39; seca fría $=44$; seca cálida $=36$ ) y 88 de lince (lluvia $=20$; seca fría $=30$; seca cálida $=38$ ). Del conjunto de heces fecales para el análisis anual se lograron identificar un total de 16 presas diferentes para la dieta del coyote y 14 para el lince (Tabla 1). Los análisis de los patrones medulares del pelo no permitieron distinguir entre las dos especies de Sylvilagus (S. floridanus y S. cunicularius) presentes en la zona de estudio, por lo que se consideraron en conjunto para los análisis posteriores.

Los mamíferos constituyeron la mayor parte en frecuencia de ocurrencia de la dieta de coyotes $(\mathrm{FO}=93.21 \%$ ) y linces $(\mathrm{FO}=81.40 \%)$. La presa con mayor frecuencia de ocurrencia fue Sylvilagus sp., en el lince (33.03\%) y en el coyote ( $25.21 \%)$, seguida por Romerolagus diazi $(27.52 \%, 23.50 \%)$. Neotomodon alstoni fue la tercera presa $(10.09 \%, 11.54 \%)$. El resto de las presas fueron encontradas en frecuencias menores. La presa que aportó mayor BTC a la dieta de ambos depredadores fue Sy/vilagus sp., con $54.58 \%$ de biomasa aportada a la dieta del coyote y $34.66 \%$ de biomasa a la del lince. El zacatuche fue la segunda presa más importante en la aportación de biomasa, con $27.18 \%$ en el lince y $\mathbf{2 3 . 7 2} \%$ en el coyote. El resto de las presas fueron poco consumidas por ambos depredadores, con valores de FO $<3 \%$ y aportando cada una $<4 \%$ a la biomasa de la dieta.

Efecto de la temporada en la dieta. Los datos de la dieta agrupados por temporada mostraron que el lince consumió en mayor proporción a Sylvilagus sp. en las temporadas de lluvia y seca fría, mientras que para la temporada seca cálida consumió a R. diazi (Figura 2). El coyote, por su parte, consumió en mayor proporción a Sylvilagus 


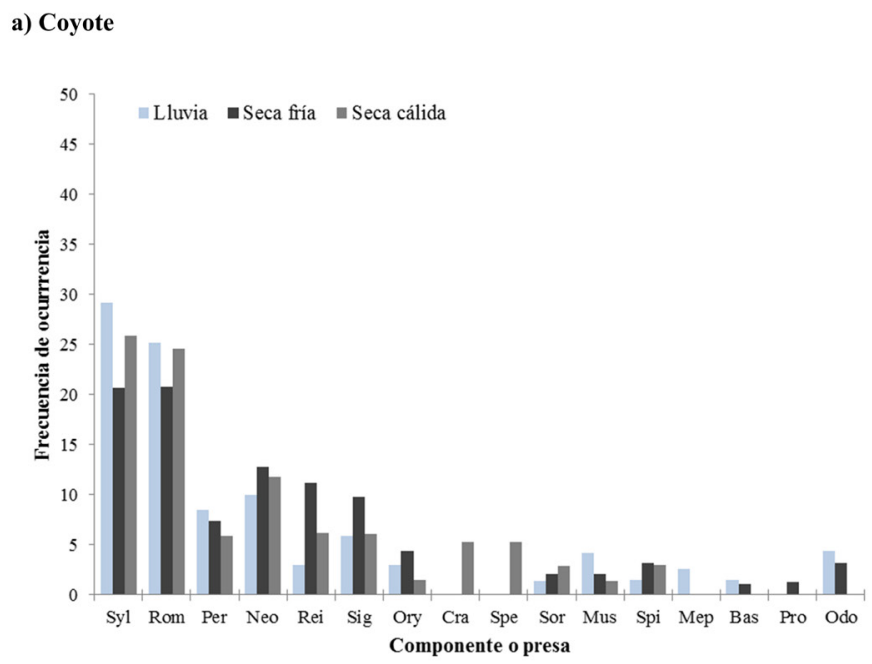

b) Lince

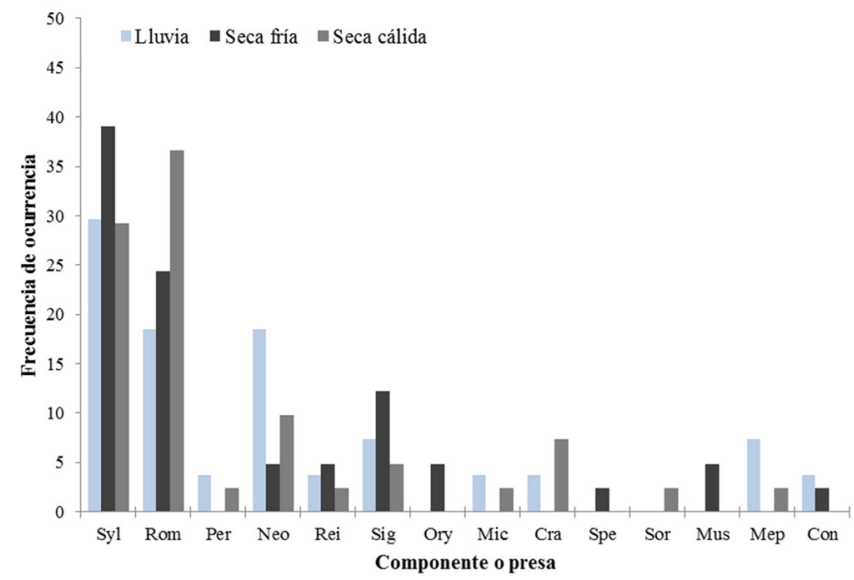

Figura 2. Gráfica de los valores de frecuencia de ocurrencia (FO) de las presas que componen la dieta del coyote y del lince durante las temporadas de lluvia, seca fría y seca cálida. Sylvilagus sp. (Syl), Romerolagus diazi (Rom), Peromyscus sp. (Per), Neotomodon alstoni (Neo), Reithrodontomys sp. (Rei), Sigmodon sp. (Sig), Oryzomys couesi (Ory), Microtus mexicanus (Mic), Cratogeomys sp. (Cra), Spermophilus sp. (Spe), Sorex sp. (Sor), Mustela frenata (Mus), Spilogale putorius (Spi), Mephitis macroura (Mep), Conepatus leuconotus (Con), Bassariscus astutus (Bas), Procyon lotor (Pro), Odocoileus virginianus (Odo).

sp. durante las temporadas seca fría y seca cálida, mientras que en la de lluvias ambos conejos fueron consumidos en altas proporciones.

La biomasa consumida tuvo un patrón muy similar a la FO, encontrando que Sylvilagus sp. fue la presa que mayor aportó a la dieta en las tres temporadas para ambos depredadores (Figura 3). Pese a estas variaciones en la dieta, la prueba de $G$ no mostró diferencias significativas en la FO de las presas entre las temporadas (coyote: $G=62.52, g . l .=46, P>0.05$; lince: $G=83.04, g . l .=36, P>0.05$ ), ni para la BTC entre las temporadas (coyote: $G=55.12, g . I .=32, P>0.05$; lince: $G=87.86, g . l .=26$, $P>0.05)$.

Abundancia relativa de presas. El conteo de heces fecales para los lagomorfos indicó que en el área de estudio la especie con mayor abundancia relativa en las tres temporadas es Romerolagus diazi con un IAR $=10.49$ heces fecales $/ \mathrm{m}^{2}$ para la temporada de lluvia, 24.38 heces fecales $/ \mathrm{m}^{2}$ para la seca fría y 16.59 heces fecales $/ \mathrm{m}^{2}$ para la seca cálida (Tabla 2). En el caso de los roedores y soricomorfos, Neotomodon 

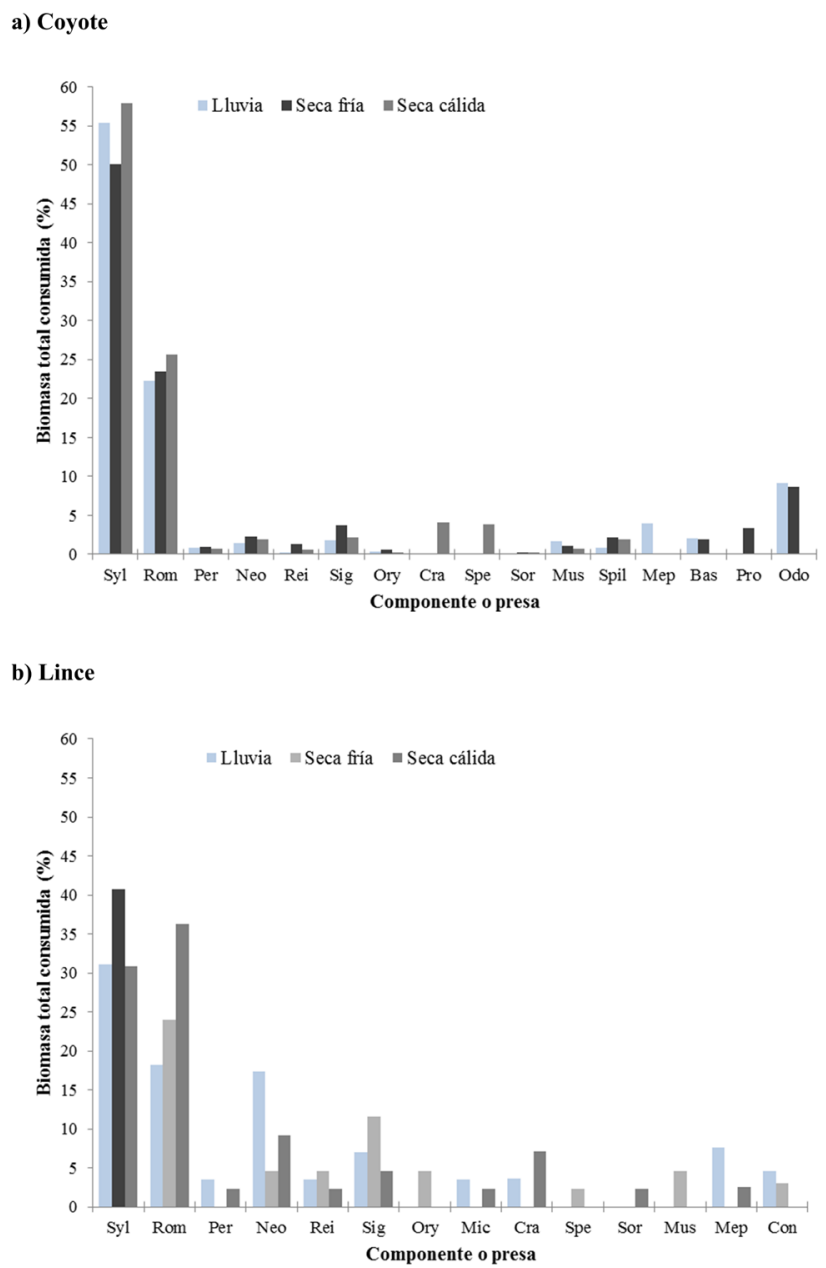

Figura 3. Gráfica de los valores de porcentaje de biomasa total consumida (BTC) en la dieta del coyote y del lince durante las temporadas de lluvia, seca fría y seca cálida. Sylvilagus sp. (Syl), Romerolagus diazi (Rom), Peromyscus sp. (Per), Neotomodon alstoni (Neo), Reithrodontomys sp. (Rei), Sigmodon sp. (Sig), Oryzomys couesi (Ory), Microtus mexicanus (Mic), Cratogeomys sp. (Cra), Spermophilus sp. (Spe), Sorex sp. (Sor), Mustela frenata (Mus), Spilogale putorius (Spi), Mephitis macroura (Mep), Conepatus leuconotus (Con), Bassariscus astutus (Bas), Procyon lotor (Pro), Odocoileus virginianus (Odo).

alstoni fue la especie más abundante en las tres temporadas, encontrando un IARRS $=0.268$ en lluvia, 0.127 en seca fría y 0.230 en seca cálida. No se capturó en las trampas ningún individuo de Microtus mexicanus, Oryzomys couesi ni de Sigmodon sp.

Análisis de selección de presas. Debido a que los análisis por temporada mostraron que la composición de la dieta en ambos depredadores no varía de manera significativa a largo del año, los análisis de selección se realizaron considerando los datos anuales para la dieta y la abundancia relativa de presas. La prueba de $X^{2}$ mostró una diferencia significativa entre la abundancia relativa de las especies presas con respecto a la biomasa consumida de éstas, tanto en la dieta del coyote (lagomorfos: $X^{2}=456.8, P<0.05$; roedores: $X^{2}=1065.2, P<0.05$ ) y del lince (lagomorfos: $X^{2}=304.1$, $P<0.05$; roedores: $X^{2}=940.6 P<0.05$ ). Los intervalos de confianza de Bonferroni demostraron que ambas especies seleccionan a Sylvilagus sp., mientras que $R$. diazi no es seleccionado por ninguno. De los roedores y soricomorfos, ambos depredadores seleccionan a Sigmodon sp. (Tabla 3). 
Tabla 1. Frecuencia de ocurrencia (FO) y porcentaje de biomasa total consumida (BTC) de cada especie presa para el análisis anual de la dieta del coyote (Canis latrans) y lince (Lynx rufus).

\begin{tabular}{|c|c|c|c|c|}
\hline \multirow[b]{2}{*}{ Especie presa } & \multicolumn{2}{|c|}{ Canis latrans $(n=119)$} & \multicolumn{2}{|c|}{ Lynx rufus $(\mathrm{n}=\mathbf{8 8})$} \\
\hline & FO (\%) & BTC (\%) & FO (\%) & BTC (\%) \\
\hline Sylvilagus sp. & 25.21 & 54.58 & 33.03 & 34.66 \\
\hline Romerolagus diazi & 23.50 & 23.72 & 27.52 & 27.18 \\
\hline Peromyscus sp. & 7.27 & 0.87 & 1.83 & 1.72 \\
\hline Neotomodon alstoni & 11.54 & 1.87 & 10.09 & 9.51 \\
\hline Reithrodontomys sp. & 6.84 & 0.69 & 3.67 & 3.44 \\
\hline Sigmodon sp. & 7.27 & 2.47 & 8.26 & 7.86 \\
\hline Oryzomys cuoesi & 2.99 & 0.39 & 1.83 & 1.73 \\
\hline Microtus mexicanus & - & - & 1.83 & 1.73 \\
\hline Cratogeomys sp. & 1.71 & 1.28 & 3.67 & 3.57 \\
\hline Spermophilus sp. & 1.71 & 1.20 & 0.92 & 0.89 \\
\hline Sorex sp. & 2.14 & 0.20 & 0.92 & 0.86 \\
\hline Mustela frenata & 2.56 & 1.14 & 1.83 & 1.76 \\
\hline Spilogale sp. & 2.56 & 1.56 & - & - \\
\hline Mephitis macroura & 0.85 & 1.50 & 2.75 & 2.83 \\
\hline Conepatus leuconotus & - & - & 1.83 & 2.27 \\
\hline Bassariscus astutus & 0.85 & 1.36 & - & - \\
\hline Procyon lotor & 0.43 & 1.03 & - & - \\
\hline Odocoileus virginianus & 2.56 & 6.13 & - & - \\
\hline
\end{tabular}

\section{Discusión}

Este es el primer estudio que aporta información sobre la selección de presas por dos de los principales carnívoros medianos de las regiones templadas del centro de México, pues los estudios anteriores únicamente se habían enfocado en la caracterización de su dieta (Romero 1993; Aranda et al. 1995; Martínez-García et al. 2014). La información sobre la selección de presas se generó con el propósito de conocer si el conejo zacatuche es consumido en función de su disponibilidad en el ambiente o si es seleccionado como presa por uno o ambos depredadores. De ser este caso, el consumo selectivo de zacatuche podría representar una presión más sobre este lagomorfo catalogado en peligro de extinción, debido a que la depredación selectiva puede provocar una disminución de las poblaciones de las presas (Vucetich et al. 2005; Gervasi et al. 2012; Ripple et al. 2013).

Nuestro estudio de la dieta del coyote y el lince en el $\mathrm{COBIOCH}$ mostró que los lagomorfos (Sylvilagus sp. y R. diazi) son las presas más utilizadas por ambas especies en términos tanto de la FO y como de por la BTC. Estos resultados son similares a estudios previos en los que únicamente se describió la dieta de estos depredadores realizados en la región del Ajusco y el Volcán Pelado en la Ciudad de México (Romero 1993; Aranda et al. 1995; Aranda et al. 2002). Sin embargo, en el caso del volcán IztaPopo se encontró que aunque los lagomorfos del género Sylvilagus fueron las presas más importantes, el zacatuche se posicionó como la cuarta presa en importancia para el lince y la quinta para el coyote (Martínez-García et al. 2014). 
Tabla 2. Índices de abundancia relativa de las principales presas (lagomorfos, roedores y soricomorfos) del coyote (Canis latrans) y el lince (Lynx rufus).

\begin{tabular}{lccc}
\multicolumn{1}{c}{ Especie } & Lluvias & Secas fría & Secas cálida \\
\hline Sylvilagus sp. & 1.23 & 2.46 & 1.33 \\
Romerolagus diazi & 10.49 & 24.38 & 16.59 \\
Peromyscus sp. & 0.026 & 0.007 & 0.005 \\
Neotomodon alstoni & 0.268 & 0.127 & 0.23 \\
Reithrodontomys sp. & 0.021 & 0.027 & 0.055 \\
Sigmodon sp. & 0 & 0 & 0.01 \\
Sorex sp. & 0 & 0.013 & 0.005 \\
\hline
\end{tabular}

La tercera presa de mayor consumo por ambos depredadores en este estudio fue el roedor N. alstoni, aunque sus valores de FO y BTC alcanzaron menos de la mitad de las obtenidas para los lagomorfos. Este resultado es distinto a los reportados anteriormente, donde N. alstoni resultó una presa de mucho menor importancia para la dieta del coyote y el lince (Romero 1993; Aranda et al. 1995; Aranda et al. 2002). Linces y coyotes comúnmente se alimentan de venado cola blanca Odocoileus virginianus (e. g. Andelt et al. 1987; McLean et al. 2005). Sin embargo, en México el venado cola blanca no ha sido reportado como una presa principal de coyotes y linces (Servín y Huxley 1993; Aranda et al. 1995; Hidalgo-Mihart et al. 2001; MartínezGarcía et al. 2014), coincidiendo con lo encontrado en este trabajo. Es posible que en el caso particular de la región del $\mathrm{COBICH}$, el bajo consumo de venado cola blanca sea reflejo de que la especie se encuentra en bajas densidades en el área (Flores-Armillas et al. 2011), lo que disminuye la posibilidad de encuentro con la especie y por tanto la importancia dentro de la dieta de coyotes y linces.

El análisis de selección de presas indicó que la composición de la dieta del lince y del coyote no está relacionada con la abundancia relativa de éstas, es decir, ambos depredadores no están consumiendo las presas en función de su disponibilidad en el ambiente como se esperaría para depredadores generalistas (Hanski et al. 1991; Lambin et al. 2000; Dell'Arte et al. 2007). En el caso de los conejos, Sylvilagus sp. fue la especie seleccionada y $R$. diazi evitada, mientras que en el caso de los ratones Sigmodon sp. y Peromyscus sp. fueron seleccionadas y N. alstoni evitada. Es importante recordar que los análisis de uso-disponibilidad se realizaron por separado para lagomorfos y roedores, por lo que es difícil la comparación de selección de presas entre ambos grupos de mamíferos. Sin embargo, el hecho de que los lagomorfos aportan el 78.3 $\%$ de la BTC para el coyote y el $61.84 \%$ para el lince, comparado con el $8.77 \%$ de roedores para el coyote y el $30.45 \%$ para el lince, muestra la mayor importancia que los lagomorfos tienen para ambos depredadores en el área de estudio y por lo tanto los resultados de uso disponibilidad.

El consumo de zacatuche en menor proporción a su disponibilidad en el ambiente y la selección de Sylvilagus sp. puede explicarse por dos razones. Las especies del género Sylvilagus sp. son de mayor tamaño y peso que el zacatuche, por lo tanto pareciera que la selección está dada por su tamaño y peso, y no por su disponibilidad en el ambiente. Esta selección de presas de mayor tamaño ha sido reportada en estudios sobre la dieta 
Tabla 3. Resultados obtenidos por medio de los intervalos de Bonferroni comparados con el uso esperado. Se muestra qué presas están siendo seleccionadas, evitadas o usadas de acuerdo a su disponibilidad en el ambiente para la dieta del coyote (Canis latrans) y del lince (Lynx rufus). Uso esperado $=\mathrm{UE}$, uso observado $=\mathrm{UO}$, límite inferior $=\mathrm{LI}$, límite superior $=\mathrm{LS}$, SE donde selección $=(+)$ y evitado $(-)$

\begin{tabular}{|c|c|c|c|c|c|c|c|c|c|c|}
\hline \multirow{3}{*}{ Especie } & \multicolumn{5}{|c|}{ Coyote } & \multicolumn{5}{|c|}{ Lince } \\
\hline & \multirow[t]{2}{*}{ UE } & \multirow[t]{2}{*}{ Uo } & \multicolumn{2}{|c|}{ Intervalos } & \multirow[t]{2}{*}{ SE } & \multirow[t]{2}{*}{ UE } & \multirow[t]{2}{*}{ Uo } & \multicolumn{2}{|c|}{ Intervalos } & \multirow[t]{2}{*}{ SE } \\
\hline & & & LI & LS & & & & LI & LS & \\
\hline Sylvilagus sp. & 0.089 & 0.518 & 0.406 & 0.629 & + & 0.088 & 0.545 & 0.408 & 0.682 & + \\
\hline Romerolagus diazi & 0.911 & 0.482 & 0.371 & 0.594 & - & 0.911 & 0.454 & 0.317 & 0.591 & - \\
\hline Neotomodon alstoni & 0.781 & 0.278 & 0.157 & 0.410 & - & 0.787 & 0.407 & 0.163 & 0.65 & - \\
\hline Sigmodon sp. & 0.013 & 0.175 & 0.071 & 0.279 & + & 0.012 & 0.333 & 0.099 & 0.566 & + \\
\hline Reihtrodontomys sp. & 0.128 & 0.165 & 0.064 & 0.266 & & 0.129 & 0.148 & -0.027 & 0.324 & \\
\hline Peromyscus sp. & 0.047 & 0.175 & 0.071 & 0.279 & + & 0.047 & 0.074 & -0.055 & 0.203 & \\
\hline Oryzomys couesi & 0.004 & 0.072 & 0.001 & 0.143 & & 0.000 & 0.065 & -0.052 & 0.181 & \\
\hline Microtus mexicanus & & & & & & 0.000 & 0.065 & -0.052 & 0.181 & \\
\hline Sorex sp. & 0.02 & 0.052 & -0.009 & 0.112 & & 0.022 & 0.037 & -0.056 & 0.130 & \\
\hline
\end{tabular}

del cánido Dhole (Cuon alpinus) que consume en mayor proporción ungulados de mayor tamaño como el chital (Axis axis) en comparación con los de tamaño menor (Andheria et al. 2007; Borah et al. 2009). Caso similar para los felinos se ha descrito la selección de las presas con mayor tamaño como se observó para la especie Otocolobus manul, donde las pikas (Ochotona dauurica) son consumidas con mayor frecuencia que el resto de las presas de menor tamaño (Ross et al. 2010).

La segunda causa que puede influir en que el zacatuche sea evitado y Sylvilagus sp. seleccionado es la diferencia entre los patrones de actividad de estas dos presas y los de sus depredadores (Molinari-Jobin et al. 2004). Los coyotes y los linces son principalmente crepusculares y nocturnos (Holzman et al. 1992; Elizalde-Arellano et al. 2012), y la presión de la cacería puede hacer que concentren su actividad en la noche (Kitchen et al. 2000). La selección hacia las especies de Sylvilagus encontrada podría deberse entonces a que su mayor actividad ocurre durante la noche, iniciando poco antes de la puesta del sol y terminando con la salida del sol (Lord 1961; Mech et al. 1966), traslapándose en gran parte con los horarios de mayor actividad del coyote y del lince. En contraparte, el zacatuche concentra sus actividades principalmente durante las horas posteriores al amanecer y por la tarde, antes del anochecer, y difícilmente se les puede encontrar por las noches (Cervantes et al. 1990; Solorio-Damián 2013). Esta sincronía entre las horas pico de actividad del coyote y sus principales presas, los lagomorfos S. audubonii y Lepus californicus, fue documentada en el desierto Chihuahuense (Arias-Del Razo et al. 2011).

Si bien el coyote y el lince presentaron selección hacia presas de mayor tamaño como los conejos del género Sylvilagus sp., nuestro estudio confirma que el zacatuche sí es una presa importante que aporta más del $20 \%$ de biomasa a la dieta de ambos depredadores. Debido a que el zacatuche una especie en peligro de extinción por la pérdida y el deterioro de su hábitat, es necesario determinar a través de estudios específicos el número potencial de zacatuches consumidos por los depredadores y su impacto en las poblaciones de la especie, dado que existe evidencia de una disminución generalizada de las poblaciones de conejos y liebres en el Oeste de 
Estados Unidos como consecuencia de la fuerte depredación por coyotes resultado de una expansión poblacional de este depredador (Ripple et al. 2013).

\section{Agradecimientos}

A la Comisión Nacional de Áreas Naturales Protegidas por el financiamiento parcial de la investigación a través del Programa de Monitoreo Biológico del zacatuche (Romerolagus diazi) en el Área de Protección de Flora y Fauna Corredor Biológico Chichinautzin. A la Dirección del Corredor Biológico Chichinautzin por las facilidades prestadas para realizar los monitoreos en la zona. A la Colección de Mamíferos del Centro de Investigación en Biodiversidad y Conservación (CMC) por la donación de pelo de ejemplares de roedores para conformar una colección de referencia. A los estudiantes de la Facultad de Ciencias Biológicas de la UAEM que nos apoyaron en el trabajo de campo.

\section{Literatura citada}

Ackerman, B. B., F. Lindzey, y T. Hemkerm. 1984. Cougar food habits in Southern Utah. Journal of Wildlife Management 48:47-155.

Andelt, W. F., J. G. Kie, F. F. Knowlton, y K. Cardwell. 1987. Variation in coyote diets associated with season and successional changes in vegetation. The Journal of Wildlife Management 51: 273-277.

Anderson, B. J., H. R. Akçakaya, M. B. Araújo, D. A. Fordham, E. Martinez-Meyer, W. Thuiller, Y B. W. Brook. 2009. Dynamics of range margins for metapopulations under climate change. Proceedings of the Royal Society B 276:1415-1420.

Andheria, A. P., K. U. Karanth, y N. S. Kumar. 2007. Diet and prey profiles of three sympatric large carnivores in Bandipur Tiger Reserve, India. Journal of Zoology 273:69-175.

Aranda, M. 2000. Huellas y otros rastros de los mamíferos grandes de México. Comisión Nacional para el Conocimiento y Uso de la Biodiversidad e Instituto de Ecología, A. C. Xalapa, México.

Aranda, M., N. López-Rivera, y L. López-De Buen. 1995. Hábitos alimentarios del coyote (Canis latrans) en la sierra del Ajusco, México. Acta Zoológica Mexicana 65:89-99.

Aranda, M., O. Rosas, J. de J. Ríos, y N. García. 2002. Análisis comparativo de la alimentación del gato montés (Lynx rufus) en dos diferentes ambientes de México. Acta Zoológica Mexicana 87:99-109

Arias-Del Razo, I., L. Hernández, J. W. Laundré, y O. Myers. 2011. Do predator and prey foraging activity patterns match? A study of coyotes (Canis latrans) and lagomorphs (Lepus californicus and Sylvilagus audubonii). Journal of Arid Environments 75:112-118.

Asociación Mexicana para el Estudio de los lagomorfos (AMCELA), Romero Malpica, F. J., H., Rangel Cordero, P. C. De Grammont, y A. D. Cuarón. 2008. Romerolagus diazi. En:

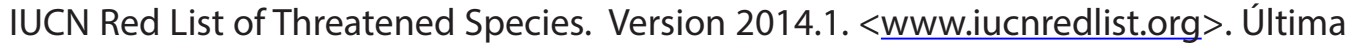
consulta 9 Septiembre 2015.

Begon, M., J. HaRPeR, y C. Townsend. 1996. Ecology: individuals, populations and communities. 3rd edition. Oxford: Blackwell Science. Malden, EE. UU.

Borah, J., K. Deka, S. Dookia, y R. Prasad Gupta. 2009. Food habits of dholes (Cuon alpinus) in Satpura Tiger Reserve, Madhya Pradesh, India. Mammalia 73:85-88.

Byers, C. R., R. K. Steinhorst, y P. R. Krausman. 1984. Clarification of a technique for analysis of utilization-availability data. Journal of Wildlife Management 48:1050-1053. 
Ceballos, G., y C. Galindo-Leal. 1984. Mamíferos silvestres de la Cuenca de México. Editorial LIMUSA. Ciudad de México, México.

Ceballos, G., y G. Oliva. 2005. Los Mamíferos Silvestres de México. Comisión Nacional Para el Conocimiento y Uso de la Biodiversidad, Fondo de Cultura Económica. Ciudad de México, México.

Cervantes, F., y J. Martínez-Vázquez. 1996. Historia natural del conejo zacatuche o teporingo (Romerolagus diazi). Pp. 29-40 en Ecología y conservación del conejo zacatuche y su hábitat (Velázquez, A., F. Romero, y J. López-Paniagua, eds.). Universidad Autónoma de México y Fondo de Cultura Económica. Ciudad de México, México.

Cervantes, F., C. Lorenzo, y S. Hoffman. 1990. Romerolagus diazi. Mammalian Species 360:1-7.

Contreras-Mac Beath, T., V. E. Anzures, A. F. Solares, J. I. Martínez, L. J. Conde, y J. C. Boyas. 2004. Conservación. Pp. 89-97 en La Diversidad Biológica en Morelos: Estudio del Estado (Contreras-Mac Beath, T., M. F. Jaramillo, y J. C. Boyas eds.). Comisión Nacional para el Conocimiento y Uso de la Biodiversidad y Universidad Autónoma del Estado de Morelos. Ciudad de México, México.

Crawford, J. A., R. G. Anthony, J. T. Forbes, y G. A. Lorton. 2010. Survival and causes of mortality for pygmy rabbits (Brachylagus idahoensis) in Oregon and Nevada. Journal of Mammalogy 91:838-847.

DelíArte, G. L., T. Laaksonen, K. Norrdahl, y E. Korpimäki. 2007. Variation in the diet composition of a generalist predator, the red fox, in relation to season and density of main prey. Acta Oecologica 31:276-281.

Drewien, R. C., S. H. Bouffard, D. D. Call, y R. A. Wonacott. 1985. The Whooping Crane crossfostering experiment: the role of animal damage control. Pp 7-13 en Proceedings of the Second Eastern Wildlife Damage Control Conference (Bromley, P. T., ed.). North Carolina, EE. UU.

Elizalde-Arellano, C., J. C. López-Vidal, L. Hernández, J. W. Laundré, F. A. Cervantes, y M. Alonso-SpILSBURy. 2012. Home range size and activity patterns of bobcats (Lynx rufus) in the Southern part of their range in the Chihuahuan desert, Mexico. The American Midland Naturalist 168:247-264.

Estes, J. A., M. T. Tinker, T. M. Williams, y D. F. Doak. 1998. Killer whale predation on sea otters linking oceanic and nearshore ecosystems. Science 282:473-476.

Festa-Bianchet, M., T. Coulson, J. M. Gaillard, J. T. Hogg, y F. Pelletier. 2006. Stochastic predation events and population persistence in bighorn sheep. Proceedings of the Royal Society B 273:1537-1543.

Flores-Armillas, V. H., S. Gallina, J. R. Barrios, V. Sánchez-Cordero, y F. J. Monrroy. 2011. Selección de hábitat por el venado cola blanca Odocoileus virginianus mexicanus (Gmelin, 1788) y su densidad poblacional en dos localidades de la región centro del Corredor Biológico Chichinautzin, Morelos, México. Therya 2:263-277.

Gervasi, S., C. Gondhalekar, D. H. Olson, y A. R. Blaustein. 2013. Host identity matters in the amphibian-Batrachochytrium dendrobatidis system: fine-scale patterns of variation in responses to a multi-host pathogen. PLoS ONE 8:e54490.

Gervasi, V., E. B. Nilsen, H. Sand, M. Panzacch, G. R. Rauset, H. C. Pedersen, J. Kindberg, P. Wabakken, B. Zimmermann, J. Odden, O. Liberg, J. E. Swenson, y J. D. C. Linnell. 2012. Predicting the potential demographic impact of predators on their prey: a comparative analysis of two carnivore-ungulate systems in Scandinavia. Journal of Animal Ecology 81:443-454.

Hall, E. R. 1981. The Mammals of North America. Segunda edición. John Wiley and Sons. New York, EE. UU. 
Hanna, E., y M. Cardillo. 2014. Island mammal extinctions are determined by interactive effects of life history, island biogeography and mesopredator suppression. Global Ecology and Biogeography 23:395-404.

Hanski, I., L. Hansson, y H. Henttonen. 1991. Specialist predators, generalist predators, and the microtines rodent cycle. Journal of Animal Ecology 60:353-367.

Hidalgo-Mihart, M. G., L. Cantú-Salazar, C. A. López-González, E. Martínez-Meyer, y A. González-Romero. 2001. Coyote (Canis latrans) food habits in a tropical deciduous forest of western Mexico. The American Midland Naturalist 146:210-216.

Holzman, S., M. J. Conroy, y J. Pickering. 1992. Home range, movements, and habitat use of coyotes in south-central Georgia. Journal Wildlife Management 56:139-146.

Kitchen, A. M., E. M. Gese, y E. R. Schauster. 2000. Changes in coyote activity patterns due to reduced exposure to human persecution. Canadian Journal of Zoology 78:853-857.

Klare, U., J. F. Kamler, y D. W. Macdonald. 2011. A comparison and critique of different scat-analysis methods for determining carnivore diets. Mammal Review 41:294-312.

Lambin, X., S. J. Petty, y J. L. Mackinnon. 2000. Cyclic dynamics in field vole populations and generalist predation. Journal of Animal Ecology 69:106-118.

Litvaitis, J. A., K. Titus, y E. M. Anderson. 1996. Measuring vertebrate use of terrestrial habitats and foods. Pp. 254-274 en Research and management techniques for wildlife and habitats. 5th ed. (Bookhout T. A., ed.). Maryland. EE. UU.

LORD, R. D. 1961. A population study of the gray fox. American Midland Naturalist 66:87-109.

Martínez-García, J. A., G. D. Menoza-Martínez, F. X. Plata, O. C. Rosas-Rosas, L. A. Tarango-Arámbula, y L. C. Bender. 2014. Use of prey by sympatric bobcat (Lynx rufus) and coyote (Canis latrans) in the lztaPopo National Park, Mexico. The Southwestern Naturalist 59:167-172.

McLean, M. L., T. S. McCaY, y M. J. Lovallo. 2005. Influence of age, sex and time of year on diet of the bobcat (Lynx rufus) in Pennsylvania. The American Midland Naturalist 153:450-453.

Mech, L. D., J. R. Tester, y D. W. Warner. 1966. Fall daytime resting habits of raccoons as determined by telemetry. Journal of Mammalogy 47:450-466.

Molinari-Jobin, A., P. Molinari, A. Loison, J. M. Gaillard, y U. Breitenmoser. 2004. Life cycle period and activity of prey influence their susceptibility to predators. Ecography 27:323-329.

Monroy-Vilchis, O., y R. Rubio-Rodríguez. 2003. Guía de identificación de mamíferos terrestres del estado de México, a través del pelo de guardia. Universidad Autónoma del Estado de México. Toluca, México.

Neu, C. W., C. R. Byers, y J. M. Peek. 1974. A technique for analysis of utilization availability data. The Journal of Wildlife Management 38:541-545.

Nissson, P. A. 2001. Predator behavior and prey density: evaluating density-dependent intraspecific interactions on predator functional responses. Journal of Animal Ecology 70:14-19.

Palomares, F. 2001. Comparison of 3 methods to estimate rabbit abundance in Mediterranean environment. Wildlife Society Bulletin 29:578-585.

Portales, G.L., P. Reyes, H. Rangel, A. Velázquez, P. Miller, S. Ellis, y A. T. Smith. 1997. International Workshop for the Conservation of Endangered Mexican Lagomorphs. IUCN/SSC Lagomorph Specialist Group and IUCN/SSC Conservation Breeding Specialist Group. Apple Valley, EE. UU.

Ripple, W. J., A. J. Wirsing, C. C. Wilmers, y M. Letnic. 2013. Widespread mesopredator effects after wolf extirpation. Biological Conservation 160:70-79.

Rizo-Aguilar, A., J. A. Guerrero, M. G. Hidalgo-Mihart, y A. González-Romero. 2015. Relationship between the abundance of the endangered volcano rabbit (Romerolagus diazi) and vegetation structure in the Sierra Chichinautzin mountain range, Mexico. Oryx 49:360-365.

Romero, R. F. 1993. Análisis de la alimentación del lince (Lynx rufus escuinapae) en el centro de México. Pp. 217-230 en Avances en el estudio de los mamíferos de México. (Medellín, R. A., y G. Ceballos, eds.). Asociación Mexicana de Mastozoología, A. C. Ciudad de México, México. 
Ross, S., B. Munkhtsog, y S. Harris. 2010. Dietary composition, plasticity, and prey selection of Pallas's cats. Journal of Mammalogy 91:811-817.

RüHE, F., T. BuRmeSter, Y M. KsinsiK. 2007. Data for estimating eaten prey masses from Eurasian lynx Lynx lynx scats in Central and East Europe. Acta Theriologica 52:317-322.

Semarnat (Secretaría de Medio Ambiente y Recursos Naturales). 2010. Norma Oficial Mexicana NOM-059SEMARNAT-2010, Protección ambiental- Especies nativas de México de flora y fauna silvestresCategorías de riesgo y especificaciones para su inclusión, exclusión o cambio- Lista de especies en riesgo. Diario Oficial de la Federación. Segunda Sección.

Servín, J., y C. Huxbey. 1993. Biología del coyote (Canis latrans) en la reserva de la biosfera "La Michilia", Durango. Pp. 197-204 en Avances en el estudio de los mamíferos de México (Medellín, R. A., y G. Ceballos, eds.). Asociación Mexicana de Mastozoología, A, C. Ciudad de México, México.

Shapira, I., H. Sultan, y U. Shanas. 2008. Agricultural farming alters predator-prey interactions in nearby natural habitats. Animal Conservation 11:1-8.

Sin, A., G. Englund, y D. Wooster. 1998. Emergent impacts of multiple predators on prey. Trends in Ecology and Evolution 13:350-355.

Sokal, R. R., y F. J. Rohlf. 1980. Introducción a la Bioestadística. Ed. Reverte. Barcelona, España.

Solorio-Damián, M. I. 2013. Descripción de la actividad crepuscular del conejo zacatuche (Romerolagus diazi) en estado silvestre en el Corredor Biológico Chichinautzin. Tesis de Licenciatura. Universidad Autónoma del Estado de Morelos. Cuernavaca, México.

StatSoft. 2007. STATISTICA (Data analysis software system). StatSoft, Inc. Tulsa, EE. UU.

Velázquez, A. 1994. Distribution and population size of Romerolagus diazi on El Pelado Volcano, México. Journal of Mammalogy 75:743-749.

Velázquez, A., y A. M. Cleef. 1993. The plant communities of the volcanoes "Tláloc" and "Pelado", México. Phytocoenologia 22:145-191.

Velázquez, A., y G. W. Heil. 1996. Habitat suitability study for the conservation of the volcano rabbit (Romerolagus diazi). Journal of Applied Ecology 33:543-554.

Velázquez, A., F. J. Romero, y J. López-Paniagua. 1996. Amplitud y utilización del hábitat del conejo zacatuche. Pp. 89-101 en Ecología y conservación del conejo zacatuche y su hábitat (Velázquez, A., F. Romero, y J. López-Paniagua eds.). Universidad Nacional Autónoma de México y Fondo de Cultura Económica. Ciudad de México, México.

Velázquez, A., A. Larrazabal, y F. Romero. 2011. Del conocimiento específico a la conservación de todos los niveles de organización biológica. El caso del zacatuche y los paisajes que denotan su hábitat. Investigación Ambiental 3:59-62.

Vucetich, J. A., D. W. Smith, y D. R. Stahler. 2005. Influence of harvest, climate and wolf predation on Yellowstone elk, 1961-2004. Oikos 111:259-270.

WeAVER, J. L. 1993. Refining the equation for interpreting prey occurrence in gray wolf scats. Journal of Wildlife Management 57:534-538.

Summited: July 2, 2015

Review: August 24, 2015

Accepted: September 23, 2015

Associated editor: Consuelo Lorenzo 
624 THERYA Vol.6(3):609-624 